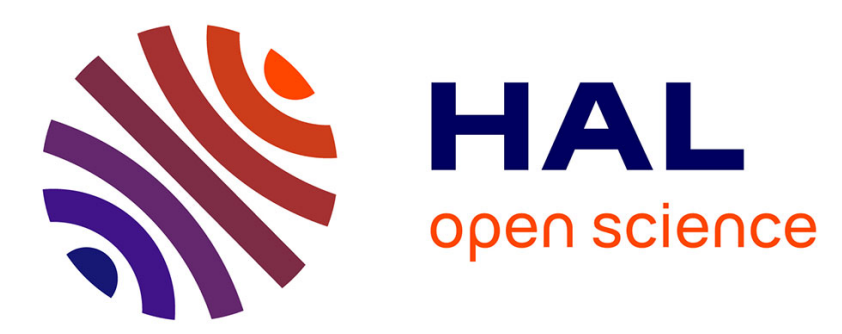

\title{
On metric embedding for boosting semantic similarity computations
}

\author{
Julien Subercaze, Christophe Gravier, Frédérique Laforest
}

\section{To cite this version:}

Julien Subercaze, Christophe Gravier, Frédérique Laforest. On metric embedding for boosting semantic similarity computations. Association of Computational Linguistics, Jul 2015, Beijing, China. hal-01166163

\section{HAL Id: hal-01166163 \\ https://hal.science/hal-01166163}

Submitted on 22 Jun 2015

HAL is a multi-disciplinary open access archive for the deposit and dissemination of scientific research documents, whether they are published or not. The documents may come from teaching and research institutions in France or abroad, or from public or private research centers.
L'archive ouverte pluridisciplinaire HAL, est destinée au dépôt et à la diffusion de documents scientifiques de niveau recherche, publiés ou non, émanant des établissements d'enseignement et de recherche français ou étrangers, des laboratoires publics ou privés. 


\title{
On metric embedding for boosting semantic similarity computations
}

\author{
Julien Subercaze, Christophe Gravier, Frederique Laforest \\ Université de Lyon, F-42023, Saint-Etienne, France, \\ CNRS, UMR5516, Laboratoire Hubert Curien, F-42000, Saint-Etienne, France, \\ Université de Saint-Etienne, Jean Monnet, F-42000, Saint-Etienne, France. \\ firstname. lastnameduniv-st-etienne. fr
}

\begin{abstract}
Computing pairwise word semantic similarity is widely used and serves as a building block in many tasks in NLP. In this paper, we explore the embedding of the shortest-path metrics from a knowledge base (Wordnet) into the Hamming hypercube, in order to enhance the computation performance. We show that, although an isometric embedding is untractable, it is possible to achieve good non-isometric embeddings. We report a speedup of three orders of magnitude for the task of computing Leacock and Chodorow ( $\mathrm{LCH})$ similarity while keeping strong correlations $(r=.819, \rho=.826)$.
\end{abstract}

\section{Introduction}

Among semantic relatedness measures, semantic similarity encodes the conceptual distance between two units of language - this goes beyond lexical ressemblance. When words are the speech units, semantic similarity is at the very core of many NLP problems. It has proven to be essential for word sense disambiguation (Mavroeidis et al., 2005; Basile et al., 2014), open domain question answering (Yih et al., 2014), and information retrieval on the Web (Varelas et al., 2005), to name a few. Two established strategies to estimate pairwise word semantic similarity includes knowledge-based and distributional semantics.

Knowledge-based approaches exploit the structure of the taxonomy ((Leacock and Chodorow, 1998; Hirst and St-Onge, 1998; Wu and Palmer, 1994)), its content ((Banerjee and Pedersen, 2002)), or both (Resnik, 1995; Lin, 1998). In the earliest applications, Wordnet-based semantic similarity played a predominant role so that semantic similarity measures reckon with information from the lexical hierarchy. It therefore ignores contextual information on word occurrences and relies on humans to encode such hierarchies - a tedious task in practice. In contrast, well-known distributional semantics strategies encode semantic similarity using the correlation of statistical observations on the occurrences of words in a textual corpora (Lin, 1998).

While providing a significant impact on a broad range of applications, (Herbelot and Ganesalingam, 2013; Lazaridou et al., 2013; Beltagy et al., 2014; Bernardi et al., 2013; Goyal et al., 2013; Lebret et al., 2013), distributional semantics - similarly to knowledge-based strategies - struggle to process the ever-increasing size of textual corpora in a reasonable amount of time. As an answer, embedding high-dimensional distributional semantics models for words into low-dimensional spaces (henceforth word embedding (Collobert and Weston, 2008)) has emerged as a popular method. Word embedding utilizes deep learning to learn a real-valued vector representation of words so that any vector distance - usually the cosine similarity - encodes the word-to-word semantic similarity. Although word embedding was successfully applied for several NLP tasks (Hermann et al., 2014; Andreas and Klein, 2014; Clinchant and Perronnin, 2013; Xu et al., 2014; Li and Liu, 2014; Goyal et al., 2013), it implies a slow training phase - measured in days (Collobert and Weston, 2008; Mnih and Kavukcuoglu, 2013; Mikolov et al., 2013), though re-embedding words seems promising (Labutov and Lipson, 2013). There is another usually under-considered issue: the tractability of the pairwise similarity computation in the vector space for large volume of data. Despite these limitations, the current enthusiasm for word embedding certainly echoes the need for lightning fast word-to-word semantic similarity computation.

In this context, it is surprising that embedding semantic similarity of words in low dimensional 
spaces for knowledge-based approaches is understudied. This oversight may well condemn the word-to-word semantic similarity task to remain corpus-dependant - i.e. ignoring the background knowledge provided by a lexical hierarchy.

In this paper, we propose an embedding of knowledge base semantic similarity based on the shortest path metric (Leacock and Chodorow, 1998), into the Hamming hypercube of size $n$ (the size of targeted binary codes). The Leacock and Chodorow semantic similarity is one of the most meaningful measure. It yields the second rank for highest correlation with the data collected by (Miller and Charles, 1991), and the first one within edge centric approaches, as shown by (Seco et al., 2004). This method is only surpassed by the information theoretic based similarity from (Jiang and Conrath, 1997). A second study present similar result (Budanitsky and Hirst, 2006), while a third one ranks this similarity measure at the first rank for precision in paraphrase identification (Mihalcea et al., 2006).

The hypercube embedding technique benefits from the execution of Hamming distance within a few cycles on modern CPUs. This allows the computation of several millions distances per second. Multi-index techniques allows the very fast computation of top-k queries (Norouzi et al., 2012) on the Hamming space. However, the dimension of the hypercube (i.e. the number of bits used to represent an element) should obey the threshold of few CPU words $(64,128 \ldots$, bits) to maintain such efficiency (Heo et al., 2012).

An isometric embedding requires a excessively high number of dimensions to be feasible. However, in this paper we show that practical embeddings exist and present a method to construct them. The best embedding presents very strong correlations $(r=.819, \rho=.829)$ with the Leacock \& Chodorow similarity measure (LCH in the rest of this paper). Our experiments against the state-of-the art implementation including caching techniques show that performance is increased by up to three orders of magnitude.

\section{Shortest path metric embedding}

Let us first introduce few notations. We denote $H_{2}^{n}$ as an n-dimensional hypercube whose nodes are labeled by the $2^{n}$ binary n-tuples. The nodes are adjacent if and only if their corresponding n-tuples differ in exactly one position, i.e. their Hamming distance $\left(\ell_{1}\right)$ is equal to one. In what follows, $Q^{n}$ denotes the metric space composed of $H_{2}^{n}$ with $\ell_{1}$.

We tackle the following problem: We aim at defining a function $f$ that maps every node $w$ of the taxonomy (Wordnet for Leacock \& Chodorow) into $Q^{n}$ so that for every pair of nodes: $\forall\left(w_{i}, w_{j}\right), d\left(w_{i}, w_{j}\right)=\lambda \cdot \ell_{1}\left(f\left(w_{i}\right), f\left(w_{j}\right)\right)$, where $\lambda$ is a scalar. For practical purposes, the construction of the mapping should also be reasonable in terms of time complexity.

Theoretical limitations Wordnet with its hypernym relation forms a partially ordered set (poset). The first approach is to perform an isometric embedding from the poset with shortest path distance into the Hamming hypercube. Such a mapping would exactly preserve the original distance in the embedding. As proven by (Deza and Laurent, 1997), poset lattices, with their shortest path metric, can be isometrically embedded into the hypercube, but the embedding requires $2^{n}$ dimensions. The resulting embedding would not fit in the memory of any existing computer, for a lattice having more than 60 nodes. Using Wordnet, with tens of thousands synsets, this embedding is untractable. The bound given by Deza et al. is not tight, however it would require a more than severe improvement to be of any practical interest.

Tree embedding To reduce the dimensionality, we weaken the lattice into a tree. We build a tree from the Wordnet's Hyponyms/Hypernyms poset by cutting 1,300 links, which correspond to roughly one percent of the edges in the original lattice. The nature of the cut to be performed can be subject to discussion. In this preliminary research, we used a simple approach. Since hypernyms are ordered, we decided to preserve only the first hypernym - semantically more relevant, or at least statistically - and to cut edges to other hypernyms.

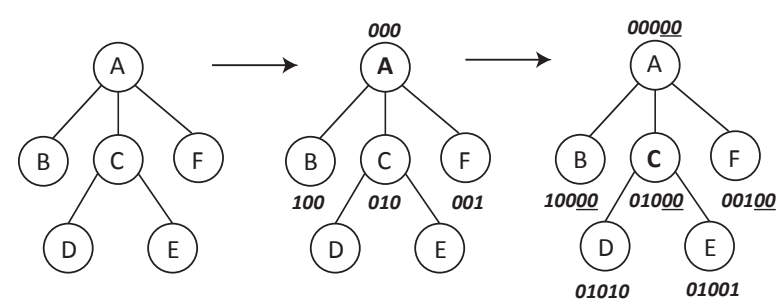

Figure 1: Construction of isometric embedding on a sample tree. For this six nodes tree, the embedding requires five bits. 
Our experiments in Table 1 shows that using the obtained tree instead of the lattice keeps a high correlation $(r=.919, \rho=.931)$ with the original LCH distance, thus validating the approach.

(Wilkeit, 1990) showed that any k-ary tree of size $n$ can be embedded into $Q^{n-1}$. We give an isometric embedding algorithm, which is linear in time and space, exhibiting a much better time complexity than Winkler's generic approach for graphs, running in $O\left(n^{5}\right)$ (Winkler, 1984). Starting with an empty binary signature, the algorithm is the following: at each step of a depth-first preorder traversal: if the node has $k$ children, we set the signature for the $i$-th child by appending $k$ zeroes to the parent's signature and by setting the $i$-th of the $k$ bits to one. An example is given in Figure 1. However, when using real-world datasets such as Wordnet, the embedding still requires several thousands of bits to represent a node. This dimension reduction to tens of kilobits per node remains far from our goal of several CPU words, and calls for a task-specific approach.

Looking at the construction of the isometric embedding, the large dimension results from the appending of bits to all nodes in the tree. This results in a large number of bits that are rarely set to one. At the opposite, the optimal embedding in terms of dimension is given by the approach of (Chen and Stallmann, 1995) that assigns gray codes to each node. However, the embedding is not isometric and introduces a very large error. As shown in Table 1, this approach gives the most compact embedding with $\left\lceil\log _{2}(87,000)\right\rceil=17$ bits, but leads to poor correlations ( $r=.235$ and $\rho=.186$ ).

An exhaustive search is also out of reach: for a fixed dimension $n$ and $r$ nodes in the tree, the number of combinations $C$ is given by:

$$
C=\frac{\left(2^{n}\right) !}{(n-r) !}
$$

Even with the smallest value of $n=17$ and $r=$ 87,000 , we have $C>10^{10,000}$. With $n=64$, to align to a CPU word, $C>10^{100,000}$.

\section{Non-isometric Embedding}

Our approach is a trade-off between the isometric embedding and the pre-order gray code solution. When designing our algorithm, we had to decide which tree distance we will preserve, either between parent and children, or among siblings.

Therefore, we take into account the nature of the tree that we aim to embed into the hypercube. Let
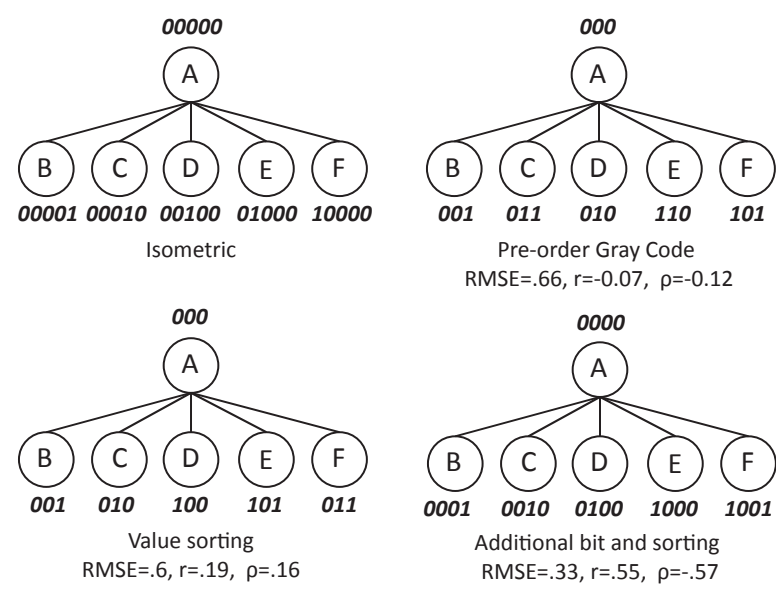

Figure 2: Approaches to reduce the tree embedding dimensions.

first analyse the characteristics of the tree obtained from the cut. The tree has an average branching factor of 4.9, with a standard deviation of 14 and $96 \%$ of the nodes have a branching factor lesser than 20. At the opposite, the depth is very stable with an average of 8.5, a standard deviation of 2 , and a maximum of 18 . Consequently, we decide to preserve the parent-children distance over the very unstable siblings distance. To lower the dimensions, we aim at allocating less than $k$ bits for a node with $k$ children, thus avoiding the signature extension taking place for every node in the isometric approach. Our approach uses the following principles.

Branch inheritance: each node inherits the signature from its father, but contrary to isometric embedding, the signature extension does not apply to all the nodes in the tree. This guarantees the compactness of the structure.

Parentship preservation: when allocating less bits than required for the isometric embedding, we introduce an error. Our allocation favours as much as possible the parentship distance at the expense of the sibling distance. As a first allocation, for a node with $k$ children, we allocate $\left\lceil\log _{2}(k+1)\right\rceil$ bits for the signatures, in order to guarantee the unicity of the signature. Each child node is assigned a signature extension using a gray code generation on the $\left\lceil\log _{2}(k+1)\right\rceil$ bits. The parent node simply extends its signature with $\left\lceil\log _{2}(k+1)\right\rceil$ zeroes, which is much more compact than the $k$ bits from the isometric embedding algorithm.

Word alignment: The two previous techniques give a compact embedding for low-depth trees, which is the case of Wordnet. The dimension $D$ 
of the embedding is not necessarily aligned to a CPU word size $W: k W \leq D \leq(k+1) W$. We want to exploit the potential $(k+1) W-D$ bits that are unused but still processed by the CPU. For this purpose we rank the nodes along a value $v(i), i \in N$ to decide which nodes are allowed to use extra bits. Since our approach favours parent/child distance, we want to allow additional bits for nodes that are both close to the root and the head of a large branch. To balance the two values, we use the following formula: $v(i)=\left(\max _{\text {depth }}-\operatorname{depth}(i)\right) \cdot \log \left(\operatorname{size}_{\text {branch }}(i)\right)$ We therefore enable our approach to take full advantage of the otherwise unused bits.

In order to enhance the quality of the embedding, we also introduce two potential optimizations:

The first is called Children-sorting: we allocate a better preserving signature to children having larger descents. A better signature is among the available the $2^{\left\lceil\log _{2}(k+1)\right\rceil}$ available, the one that reduces the error with the parent node. We rank the children by the size of their descent and assign the signatures accordingly.

The second optimization is named Valuesorting and is depicted in Figure 2. Among the $2^{\left\lceil\log _{2}(k+1)\right\rceil}$ available signatures, only $k+1$ will be assigned (one for the parent and $k$ for the children). For instance in the case of 5 children as depicted in Figure 2, we allocate 3 bits for 6 signatures. We favor the parentship distance by selecting first the signatures where one bit differs from the parent's one.

\section{Experiments}

In this section, we run two experiments to evaluate both the soundness and the performance of our approach. In the first experiment, we test the quality of our embedding against the tree distance and the LCH similarity. The goal is to assess the soundness of our approach and to measure the correlation between the approximate embedding and the original LCH similarity.

In the second experiment we compare the computational performance of our approach against an optimized in-memory library that implements the LCH similarity.

Our algorithm called FSE for Fast Similarity Embedding, is implemented in Java and available publicly ${ }^{1}$. Our testbed is an Intel Xeon E3

\footnotetext{
${ }^{1}$ Source code, binaries and instructions to reproduce
}

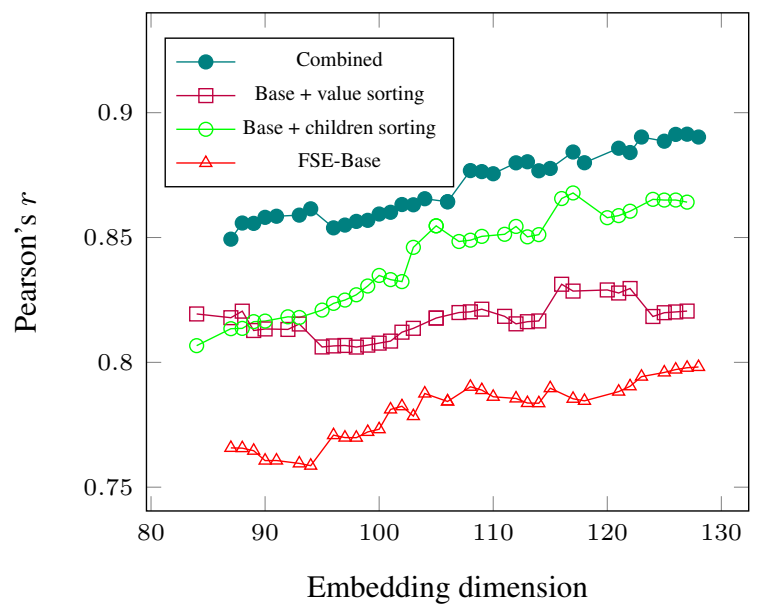

Figure 3: FSE: influence of optimizations and dimensions on the correlation over the tree distance on Wordnet.

1246v3 with 16GB of memory, a 256Go PCI Express SSD. The system runs a 64-bit Linux 3.13.0 kernel with Oracle's JDK 7u67.

The FSE algorithm is implemented in various flavours. FSE-Base denotes the basic algorithm, containing none of the optimizations detailed in the previous section. FSE-Base can be augmented with either or both of the optimizations. This latter version is denoted FSE-Best.

\subsection{Embedding}

We first measure the correlation of the embedded distance with the original tree distance, to validate the approach and to determine the gain induced by the optimizations. Figure 3 shows the influence of dimensions and optimizations on the Pearson's product moment correlation $r$. The base version reaches $r=.77$ for an embedding of dimension 128. Regarding the optimizations, children sorting is more efficient than value sorting, excepted for dimensions under 90. Finally, combined optimizations (FSE-Best) exhibit a higher correlation $(r=.89)$ than the other versions.

We then measure the correlation with the Leacock \& Chodorow similarity measure. We compare our approach to the gray codes embedding from (Chen and Stallmann, 1995) as well as the isometric embedding. We compute the correlation on 5 millions distances from the Wordnet-Core noun pairs ${ }^{2}$ (Table 1). As expected, the embed-

\footnotetext{
the experiments are available at http://demo-satin. telecom-st-etienne.fr/FSE/

${ }^{2}$ https: / / wordnet.princeton.edu/ wordnet/download/standoff/
} 


\begin{tabular}{lrrr} 
Embedding & Bits & Pearson's $r$ & Spearman's $\rho$ \\
\hline Chen et al. & 17 & .235 & .186 \\
FSE-Base & 84 & .699 & .707 \\
FSE-Best & $\mathbf{1 2 8}$ & $\mathbf{. 8 1 9}$ & $\mathbf{. 8 2 9}$ \\
Isometric & $84 \mathrm{~K}$ & .919 & .931 \\
\hline
\end{tabular}

Table 1: Correlations between $\mathrm{LCH}$, isometric embedding, and FSE for all distances on all WordnetCore noun pairs $\left(p\right.$-values $\left.\leq 10^{-14}\right)$.

\begin{tabular}{llccccc}
\hline \multirow{2}{*}{ Algorithm } & \multirow{2}{*}{ Measure } & \multicolumn{5}{c}{ Amount of pairs $(n)$} \\
\cline { 3 - 7 } & & $10^{3}$ & $10^{4}$ & $10^{5}$ & $10^{6}$ & $10^{7}$ \\
\hline WS4J & $10^{3} \cdot \mathrm{ms}$ & 0.156 & 1.196 & 11.32 & 123.89 & $1,129.3$ \\
\cline { 2 - 7 } \begin{tabular}{c} 
FSE-Best \\
\multicolumn{1}{c}{ speedup }
\end{tabular} & $\mathrm{ms}$ & 0.04 & 0.59 & 14.15 & 150.58 & 1,482 \\
\cline { 2 - 7 } & $\times 3900$ & $\times 2027$ & $\times 800$ & $\times 822$ & $\times 762$ \\
\hline
\end{tabular}

Table 2: Running time in milliseconds for pairwise similarity computations.

ding obtained using gray codes present a very low correlation with the original distance.

Similarly to the results obtained on the tree distance correlation, FSE-Best exhibits the highest scores with $r=.819$ and $\rho=.829$, not far from the theoretical bound of $r=.919$ and $\rho=.931$ for the isometric embedding of the same tree. Our approach requires 650 times less bits than the isometric one, while keeping strong guarantees on the correlation with the original LCH distance.

\subsection{Speedup}

Table 4.2 presents the computation time of the $\mathrm{LCH}$ similarity. This is computed using $\mathrm{WS} \mathrm{J}^{3}$, an efficient library that enables in-memory caching.

Because of the respective computational complexities of the Hamming distance and the shortest path algorithms, FSE unsurprisingly boosts LCH similarity computation by orders of magnitudes. When the similarity is computed on a small number of pairs (a situation of the utmost practical interest), the factor of improvement is three orders of magnitude. This factor decreases to an amount of 800 times for very large scale applications. The reason of the decrease is that WS4J caching mechanism becomes more efficient for larger numbers of comparisons. As the caching system stores shortest path between nodes, these computed values are more likely to be a subpath of another query when the number of queries grows.

\footnotetext{
${ }^{3}$ https://code.google.com/p/ws 4 j/
}

\section{Conclusion}

We proposed in this paper a novel approach based on metric embedding to boost the computation of shortest-path based similarity measures such as the one of Leacock \& Chodorow. We showed that an isometric embedding of the Wordnet's hypernym/hyponym lattice does not lead to a practical solution. To tackle this issue, we weaken the lattice structure into a tree by cutting less relevant edges. We then devised an algorithm and several optimizations to embed the tree shortest-path distance in a word-aligned number of bits. Such an embedding can be used to boost NLP core algorithms - this was demonstrated here on the computation of LCH for which our approach offers a factor of improvement of three orders of magnitude, with a very strong correlation.

\section{Acknowledgements}

This work is supported by the OpenCloudware project. OpenCloudware is funded by the French FSN (Fond national pour la Société Numérique), and is supported by Pôles Minalogic, Systematic and SCS.

\section{References}

Jacob Andreas and Dan Klein. 2014. How much do word embeddings encode about syntax. In Association for Computational Linguistics (ACL).

Satanjeev Banerjee and Ted Pedersen. 2002. An adapted lesk algorithm for word sense disambiguation using wordnet. In Computational linguistics and intelligent text processing, pages 136-145. Springer.

Pierpaolo Basile, Annalina Caputo, and Giovanni Semeraro. 2014. An enhanced lesk word sense disambiguation algorithm through a distributional semantic model. In Proceedings of COLING, pages 1591-1600.

Islam Beltagy, Katrin Erk, and Raymond Mooney. 2014. Semantic parsing using distributional semantics and probabilistic logic. Association for Computational Linguistics (ACL), page 7.

Raffaella Bernardi, Georgiana Dinu, Marco Marelli, and Marco Baroni. 2013. A relatedness benchmark to test the role of determiners in compositional distributional semantics. In Association for Computational Linguistics (ACL), pages 53-57.

Alexander Budanitsky and Graeme Hirst. 2006. Evaluating wordnet-based measures of lexical semantic relatedness. Computational Linguistics, 32(1):1347. 
Woei-Kae Chen and Matthias FM Stallmann. 1995. On embedding binary trees into hypercubes. Journal of Parallel and Distributed Computing, 24(2):132-138.

Stéphane Clinchant and Florent Perronnin. 2013. Aggregating continuous word embeddings for information retrieval. Association for Computational Linguistics (ACL).

Ronan Collobert and Jason Weston. 2008. A unified architecture for natural language processing: Deep neural networks with multitask learning. In Proceedings of the 25th international conference on Machine learning, pages 160-167. ACM.

M. Deza and M. Laurent. 1997. Geometry of Cuts and Metrics. Springer, 588 pages.

Kartik Goyal, Sujay Kumar Jauhar, Huiying Li, Mrinmaya Sachan, Shashank Srivastava, and Eduard H Hovy. 2013. A structured distributional semantic model for event co-reference. In Association for Computational Linguistics (ACL), pages 467-473.

Jae-Pil Heo, Youngwoon Lee, Junfeng He, Shih-Fu Chang, and Sung-Eui Yoon. 2012. Spherical hashing. In Computer Vision and Pattern Recognition (CVPR), 2012 IEEE Conference on, pages 2957 2964. IEEE.

Aurélie Herbelot and Mohan Ganesalingam. 2013. Measuring semantic content in distributional vectors. In Association for Computational Linguistics (ACL), pages 440-445.

Karl Moritz Hermann, Dipanjan Das, Jason Weston, and Kuzman Ganchev. 2014. Semantic frame identification with distributed word representations. In Association for Computational Linguistics (ACL).

Graeme Hirst and David St-Onge. 1998. Lexical chains as representations of context for the detection and correction of malapropisms. WordNet: An electronic lexical database, 305:305-332.

Jay J Jiang and David W Conrath. 1997. Semantic similarity based on corpus statistics and lexical taxonomy. Proceedings of the 10th Research on Computational Linguistics International Conference.

Igor Labutov and Hod Lipson. 2013. Re-embedding words. In ACL (2), pages 489-493.

Angeliki Lazaridou, Marco Marelli, Roberto Zamparelli, and Marco Baroni. 2013. Compositional-ly derived representations of morphologically complex words in distributional semantics. In Association for Computational Linguistics (ACL), pages 15171526.

Claudia Leacock and Martin Chodorow. 1998. Combining local context and wordnet similarity for word sense identification. WordNet: An electronic lexical database, 49(2):265-283.
Rémi Lebret, Joël Legrand, and Ronan Collobert. 2013. Is Deep Learning Really Necessary for Word Embeddings? Technical report, Idiap.

Chen Li and Yang Liu. 2014. Improving text normalization via unsupervised model and discriminative reranking. Association for Computational Linguistics $(A C L)$, page 86.

Dekang Lin. 1998. An information-theoretic definition of similarity. In ICML, volume 98, pages $296-$ 304.

Dimitrios Mavroeidis, George Tsatsaronis, Michalis Vazirgiannis, Martin Theobald, and Gerhard Weikum. 2005. Word sense disambiguation for exploiting hierarchical thesauri in text classification. In Knowledge Discovery in Databases: PKDD 2005, pages 181-192. Springer.

Rada Mihalcea, Courtney Corley, and Carlo Strapparava. 2006. Corpus-based and knowledge-based measures of text semantic similarity. In $A A A I$, volume 6 , pages $775-780$.

Tomas Mikolov, Kai Chenand, Greg Corradoand, and Jeffrey Dean. 2013. Efficient estimation of word representations in vector space. In Proceedings of the International Conference on Learning Representations.

George A Miller and Walter G Charles. 1991. Contextual correlates of semantic similarity. Language and cognitive processes, 6(1):1-28.

Andriy Mnih and Koray Kavukcuoglu. 2013. Learning word embeddings efficiently with noise-contrastive estimation. In C.J.C. Burges, L. Bottou, M. Welling, Z. Ghahramani, and K.Q. Weinberger, editors, $A d$ vances in Neural Information Processing Systems 26, pages 2265-2273. Curran Associates, Inc.

Mohammad Norouzi, Ali Punjani, and David J Fleet. 2012. Fast search in hamming space with multiindex hashing. In Computer Vision and Pattern Recognition (CVPR), 2012 IEEE Conference on, pages 3108-3115. IEEE.

Philip Resnik. 1995. Using information content to evaluate semantic similarity in a taxonomy. In Proceedings of the 14th International Joint Conference on Artificial Intelligence - Volume 1, IJCAI'95, pages 448-453, San Francisco, CA, USA. Morgan Kaufmann Publishers Inc.

Nuno Seco, Tony Veale, and Jer Hayes. 2004. An intrinsic information content metric for semantic similarity in wordnet. In ECAI, volume 16, page 1089.

Giannis Varelas, Epimenidis Voutsakis, Paraskevi Raftopoulou, Euripides GM Petrakis, and Evangelos E Milios. 2005. Semantic similarity methods in wordnet and their application to information retrieval on the web. In Proceedings of the 7th annual ACM international workshop on Web information and data management, pages 10-16. ACM. 
Elke Wilkeit. 1990. Isometric embeddings in hamming graphs. Journal of Combinatorial Theory, Series $B, 50(2): 179-197$.

Peter M Winkler. 1984. Isometric embedding in products of complete graphs. Discrete Applied Mathematics, 7(2):221-225.

Zhibiao Wu and Martha Palmer. 1994. Verbs semantics and lexical selection. In Proceedings of the 32nd annual meeting on Association for Computational Linguistics, pages 133-138. Association for Computational Linguistics.

Liheng Xu, Kang Liu, Siwei Lai, and Jun Zhao. 2014. Product feature mining: Semantic clues versus syntactic constituents. In Association for Computational Linguistics (ACL), pages 336-346.

Wen-tau Yih, Xiaodong He, and Christopher Meek. 2014. Semantic parsing for single-relation question answering. In Proceedings of ACL. 